\title{
Unique shape determination of icosahedrally symmetric particles labeled with a strong scatterer
}

from solution $x$-ray scattering experiments

Youngha Hwang, Purdue University

The radial components of the particle density function can't be determined uniquely because the scattering intensity gives only information about the radial autocorrelation function of the particle density function. For each order(or quantum number), orthogonal linear combinations of the corresponding radial particle density function of different states lead to identical scattering intensity. However, in case of icosahedral symmetry, the above non-uniqueness simplifies to sign ambiguity of the radial particle density functions for order less than thirty, due to only one state of the icosahedral harmonics.

Strong scatterer experiment is motivated by isomorphous replacement method in macromolecular single-crystal x-ray diffraction. Two patterns of scattering are compared. One pattern is from a crystal made from the molecule of interest, and the other one is from a crystal made from a chemical derivative of the molecule of interest where the derivative is the addition of heavy metal atoms with many electrons. Without any constraint of an atomic-size volume in single-crystal diffraction, the number of added electrons can be much greater in solution scattering. Therefore they are strong scatterers and ideally do not alter the structure of the molecule. Experimental techniques for adding organo-metallic gold clusters were already developed.

The symmetry of the array of the strong scatterers is assumed to be a subgroup of the symmetry of the particle of interest for the experiment. An icosahedrally symmetric impulse model was developed for the strong scatterer or the decorator. However, the decorator cannot be infinitesimal, so a spherical blob model of a constant electronic density is proposed here. The necessary number of electrons is studied so that the decorator should be effective against noises. Other considerations are made for the realistic design of the strong scatterer experiment such as a perturbation model of the decorator locations and a probabilistic model of partial occupancy of the decorators because the array does not need to be completely occupied. Numerical analysis shows that the sign ambiguity of the radial particle density function can be resolved with the proposed decorator model. 